\title{
Factores que Influyen en el Emprendimiento y su Incidencia en el Desarrollo Económico del Ecuador
}

\author{
Factors that Influence Entrepreneurship and its Impact on the Economic \\ Development of Ecuador \\ * Mercedes Moreira Menéndez ${ }^{1}$, Inés De La Luz Bajaña Mendieta1 ${ }^{1}$, Bolívar Roberto \\ Pico Saltos ${ }^{1}$, Gleiston Ciceron Guerrero Ulloa ${ }^{1}$, José Vicente Villlarroel Bastidas ${ }^{1}$ \\ ${ }^{1}$ Universidad Técnica Estatal de Quevedo \\ *mmoreira@uteq.edu.ec
}

Fecha de recepción: 10/12/2017

Fecha de aceptación: 03/03/2018

Publicado: 29/06/2018

\section{Resumen}

El emprendimiento ha cobrado interés para las escuelas de negocios desde la década de los ochenta debido a la relación con la creación y dirección de empresas, entre otras razones. El emprendimiento es un campo vasto que involucra diferentes tópicos como financiación del emprendimiento, características del emprendedor, emprendimiento corporativo, empresas de familia, reconocimiento de oportunidades, aprendizaje y emprendimiento social, entre otros.

Hoy en día es imposible ignorar la importancia que tienen los emprendedores en la generación de riqueza en el país y en el mundo, lo cual amerita que se realice un estudio en detalle de su conducta y de los factores que coadyuvan a que los mismos tengan éxito en la gestión de sus emprendimientos.

El análisis económico afirma que los emprendedores tienen una gran influencia sobre el crecimiento económico. Sus decisiones sobre la inversión y sobre la producción favorecen 
la creación de riqueza y fomentan el bienestar social. El principal objetivo de este trabajo es analizar la relación entre emprendedores y crecimiento económico, considerando los factores que favorecen el emprendimiento. Para ello, el trabajo se divide en dos partes. Una teórica en la que se exponen los diferentes aspectos relacionados con los emprendedores según diversos autores. Y empírica, analizando la relación entre emprendimiento y crecimiento económico.

Palabras Clave: emprendimiento, crecimiento económico, factores influyen el emprendimiento.

\begin{abstract}
Entrepreneurship has gained interest in business schools since the 1980s because of the relationship with business creation and management, among other reasons. Entrepreneurship is a vast field that involves different topics such as entrepreneurship financing, characteristics of the entrepreneur, corporate entrepreneurship, family companies, recognition of opportunities, learning and social entrepreneurship, among others.

Today it is impossible to ignore the importance of entrepreneurs in generating wealth in the country and in the world, which requires a detailed study of their behavior and the factors that contribute to their success In the management of their enterprises.

Economic analysis states that entrepreneurs have a great influence on economic growth. Their decisions about investment and production favor wealth creation and promote social welfare. The main objective of this paper is to analyze the relationship between
\end{abstract}


entrepreneurs and economic growth, considering the factors that favor entrepreneurship. For this, the work is divided into two parts. A theoretical in which the different aspects related to the entrepreneurs are exposed according to diverse authors, and empirical, analyzing the relationship between entrepreneurship and economic growth.

Keywords: Entrepreneurship, economic growth, factors influence entrepreneurship.

\section{Introducción}

Dentro de los sectores económicos-productivos es importante establecer empresas e instituciones que fomenten la creación de empleos y dé valor a la sociedad, incluidos: empleados, dirigentes, inversionistas, así como el gobierno y consumidor final. Es por ello que la creación y dirección de empresas es de vital importancia para el sostén de los desarrollos de los países y las regiones económicas que imperan actualmente (Moreno, 2009). El emprendimiento entendido de forma amplia como el desarrollo de una idea o negocio, principalmente en el ámbito empresarial, es hoy sin duda un fenómeno de gran interés y especialmente si éste es innovador. Los países más competitivos y las economías más desarrolladas se distinguen por contar con un tejido productivo que tiene su origen en un emprendimiento que podemos llamar inteligente, ya que está basado en uso innovador del conocimiento y la creatividad, y en tecnologías avanzadas, que muestra además gran capacidad de mercado y de internacionalización (Lederma Daniel, 2014).

En América Latina y el Caribe, las actividades emprendedoras y en especial la figura del emprendedor, han cobrado una especial relevancia sobre todo en la última década con el surgimiento de un sinnúmero de iniciativas destinadas a la promoción y ayuda a las 
actividades de creación de nuevas empresas. Las personas que inician un emprendimiento representan un $18 \%$ de la población económicamente activa (10\% en Panamá y $30 \%$ en Bolivia) y quienes ya están establecidos un 10\% (3,3\% en Guatemala y 19\% en Bolivia). El comercio cubre un $64 \%$ de emprendimientos iniciales y $56 \%$ de establecidos, lo que hace la región similar a Asia y distante como 20 puntos porcentuales de EEUU y Europa, (GEM, 2015).

Existen países como Colombia, que busca implementar la cultura del emprendimiento en las instituciones educativas desde el nivel básico primaria, hasta la formación universitaria. En Chile, el Gobierno ha implementado una serie de políticas públicas que apunten a incentivar el emprendimiento. A medida que los países son más desarrollados, en promedio tienden a presentar menores actividades de comercio, como el caso de Europa y Estados Unidos. Sin embargo, es importante remarcar que la principal potencia emergente, China, tiene los mayores porcentajes de actividad emprendedora de comercio (Herrera, 2012).

En el Ecuador, el gobierno a través de la Secretaria Nacional de Planificación (SENPLADES), instrumenta como política de Estado el Plan del Buen Vivir (2008-2013), que hoy se ejecuta el Plan 2013-2017 con el fin de promocionar emprendimientos exitosos, generadores de empleo. En el año 2014, Ecuador obtuvo un índice de Actividad Emprendedora Temprana (TEA) de 32,6\%, es decir, que uno de cada tres adultos había iniciado los trámites para establecer un negocio, o poseía uno cuya antigüedad no superaba los cuarenta y dos meses. Sin embargo, la TEA del 2014 registra una leve reducción, que en el 2013 se ubicó en el 36\%, en el 2012 fue de 26,6\%, y en el 2010 fue 21,3\% (GEM, 2015).

A pesar de existir un alto porcentaje de emprendimiento en Ecuador, el índice de 
subempleo se ubicó en 17,1\% a marzo de 2016, a marzo de 2015 se ubicó en 13,37\% y al 12,63\% a marzo de 2014 (ENEMDU, 2016, Marzo). Augusto de la Torre, Economista en Jefe del Banco Mundial para América Latina y el Caribe, aclara que un emprendedor exitoso es aquel que transforma las ideas en empresas comerciales rentables, un proceso que requiere la capacidad de innovar, introducir nuevos productos y explorar nuevos mercados.

Este artículo tiene como objetivo identificar los factores que fomentan o dificultan la generación de nuevos emprendimientos y su incidencia en la relación existente entre el emprendimiento, crecimiento económico, tasa de empleo y tasa de subempleo en el Ecuador.

\section{Desarrollo}

\section{Características del emprendimiento}

Drucker (1997), define al entrepreneur como aquel empresario que es innovador, destaca que aunque alguien abra un pequeño negocio y corra riesgos, eso no quiere decir que sea innovador y represente un emprendimiento. Traduce la acción empresarial como una conducta más que un rasgo del carácter (Varela, 2001). Hace diferencia entre un pequeño negocio tradicional y un verdadero emprendimiento; el cual es aquel que genera cambios en los mercados. No todo negocio es un emprendimiento innovador.

Según lo estudiado por (Hisrich, P.; Peters, M.P; Shepherd, D.A, 2005), la definición de lo que es un emprendimiento se puede dar desde diferentes puntos de vista:

Cienc. Soc. y Econ. UTEQ. 2018. 2(1): 1-22 5 
- Desde la visión del comportamiento: es el que emprende la iniciativa, puede organizar y reorganizar los mecanismos sociales y económicos para convertir en recursos y situaciones en algo práctico, y la aceptación al riesgo por fracasar.

- Desde la visión economista: quien convierte recursos, trabajo, materiales y otros insumos, en establecer mejoras para modificar y aumentar su valor económico, y en complemento a esto, introduce cambios, innovaciones y nuevas estructuras socioeconómicas.

- Desde la visión psicológica: quien está impulsado por la fuerza de alcanzar o lograr un ideal, de experimentar, de conseguir (o en su caso), la capacidad de escapar o no depender de otros.

Según (Reynolds, 2005), distingue entre emprendimiento por oportunidad y emprendimiento por necesidad. El primero, guiado por la identificación de un negocio potencialmente atractivo, tiende a generar más empleo, a exportar en mayor medida y a crear nuevos nichos de mercado. Por el contrario, el segundo se da cuando los individuos se ven forzados a emprender por las circunstancias, para encontrar mejores maneras de subsistir.

Toda decisión emprendedora suele estar motivada por las expectativas y los estándares de vida de la población, de ahí que en cada tipo de economía se observen más o menos emprendedores por necesidad y/o por oportunidad. En Ecuador, en el año 2015 el 69\% de la TEA corresponde a emprendimientos por oportunidad y el $31 \%$ por necesidad. La TEA urbana por oportunidad se reduce un poco y se observa en cambio un aumento de esta motivación para la TEA rural, manteniéndose la TEA rural por necesidad. 


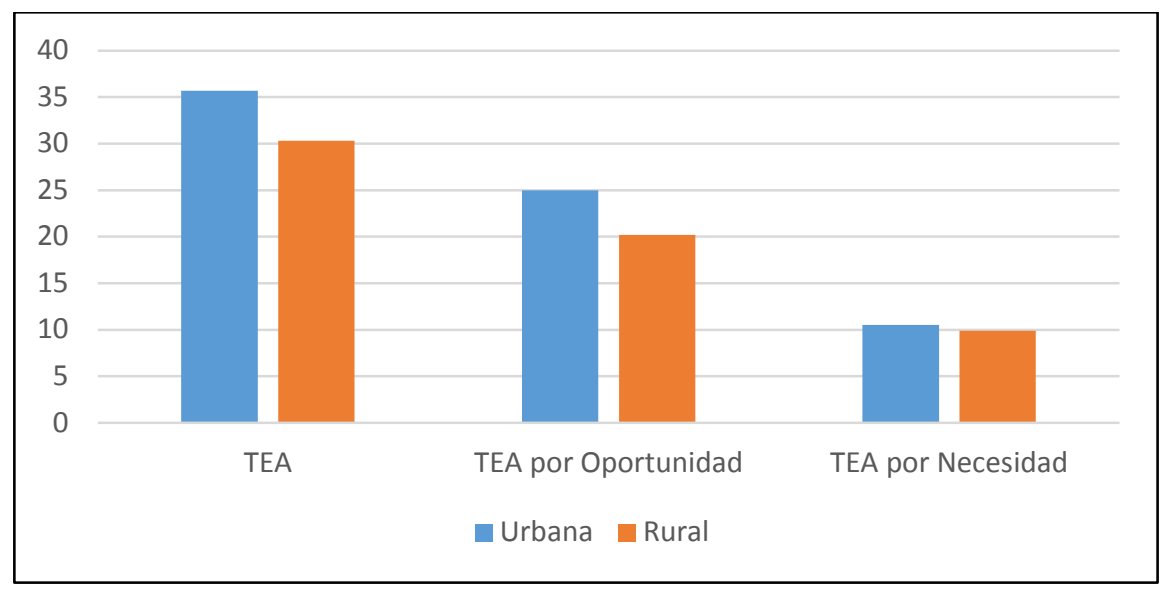

Figura 1. Actividad Emprendedora Temprana (TEA) por zona y motivación. GEM Ecuador 2015

En general, el emprendedor trata de identificar una oportunidad a partir de la cual obtener una ventaja competitiva. Esta combinación deriva en empresas orientadas al crecimiento que crean valor para sus clientes y riqueza para sus fundadores. Es una opción de presente y de futuro para nuestros jóvenes. Principalmente, supone una oportunidad de desarrollar su creatividad, talento y capacidad de innovación, pero siempre siendo conscientes de que para ello deben contar con una adecuada formación: educación y entrenamiento.

\section{Factores que influyen en el emprendimiento}

Con respecto a los factores que inciden en el éxito de los emprendimientos existe cierta subjetividad, ya que no se encuentra una identificación universal o generalizable, si no que se encuadra dentro de cada contexto o realidad específica identificándose un conjunto de factores internos y externos. Dentro de los factores externos se encuentran: situación financiera, condiciones económicas, competencia, regulación tecnológica, situación 
política, mercado laboral; y entre los factores internos planteados se encuentran: modelo de administración, actividades de marketing, gestión del talento humano y características de los individuos. Considerando lo expuesto por (Lorca, 2013), quien menciona que los factores internos determinan el éxito, mientras que los factores externos determinan el fracaso, se establece que los factores externos no son controlados por el emprendedor, mientras que los factores internos si dependen del emprendedor y es por este hecho que el autor los considera arbitrariamente como factores de éxito o fracaso.

En este sentido, Ramírez (2009) manifiesta que cuando se inicia un emprendimiento comienza una aventura llena de factores que pueden ser: personales, de mercado, y de un contexto cambiante que influye en el éxito de los emprendimientos.

El estudio realizado por Global Entrepreneurship Monitor incluye factores del entorno, que proveen las condiciones para el desarrollo de la actividad emprendedora, tanto en sus etapas tempranas como para su sostenibilidad, denominadas condiciones o factores marco para el emprendimiento, y que están en correspondencia con la etapa de desarrollo económico en que se encuentra el país. El estudio que se realizó en Ecuador en el año 2015, se identifican los siguientes factores de emprendimiento: apoyo financiero, capacidad para emprender, programas de gobierno, políticas gubernamentales, educación y entrenamiento, apertura del mercado, y normas sociales y culturales. 


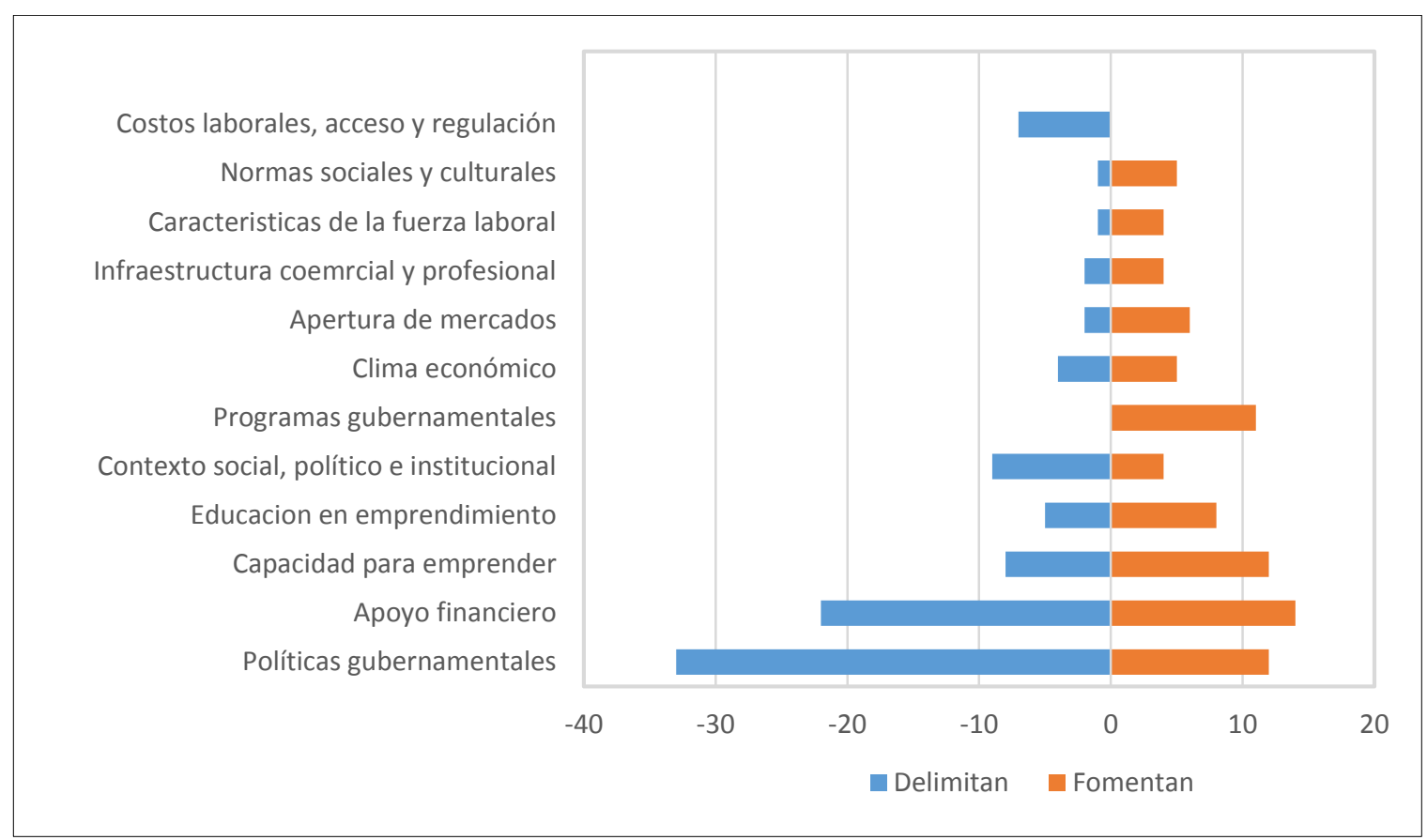

Figura 2. Factores que fomentan y delimitan el emprendimiento en Ecuador. GEM Ecuador 2015

Los factores que fomentan la actividad emprendedora en Ecuador, podrían distinguirse como fortalezas: capacidad para emprender, programas gubernamentales, educación y entrenamiento, apertura del mercado interno, clima económico e infraestructura profesional y comercial, características de la fuerza de trabajo es un factor que contribuye con el emprendimiento, sin embargo los motivos no reflejan necesariamente fortalezas del entorno. Entre las debilidades se contarían los siguientes factores: políticas de gobierno, apoyo financiero, contexto social, político e institucional, legislación laboral - costos laborales, acceso y legislación.

GEDI (2014-2015), analiza las interacciones entre las actitudes, habilidades y aspiraciones emprendedoras, así como las instituciones y variables del entorno. El siguiente cuadro muestra los índices en el Ecuador. 
Tabla 1.

Índice de actitudes, habilidades y aspiraciones emprendedoras en Ecuador

\begin{tabular}{|c|c|c|c|}
\hline Subíndice & Pilares & 2014 & 2015 \\
\hline $\begin{array}{l}\text { Actitudes } \\
\text { Emprendedoras }\end{array}$ & $\begin{array}{l}\text { Percepción de habilidades } \\
\text { Habilidades para iniciar empresas } \\
\text { Aceptación del riesgo } \\
\text { Networking } \\
\text { Apoyo cultural }\end{array}$ & $\begin{array}{ll}0,66 & (2) \\
0,63 & (3) \\
0,16 & (10) \\
0,32 & (6) \\
0,37 & (5)\end{array}$ & $\begin{array}{ll}0,67 & (1) \\
0,62 & (2) \\
0,27 & (8) \\
0,35 & (5) \\
0,35 & (6)\end{array}$ \\
\hline $\begin{array}{l}\text { Habilidad } \\
\text { Emprendedora }\end{array}$ & $\begin{array}{l}\text { Emprendimiento por oportunidad } \\
\text { Absorción tecnológica } \\
\text { Capital humano } \\
\text { Competencia }\end{array}$ & $\begin{array}{ll}0,22 & (8) \\
0,20 & (9) \\
0,23 & (7) \\
0,46 & (4)\end{array}$ & $\begin{array}{ll}0,22 & (9) \\
0,17 & \\
1) \\
0,27 \\
0,57\end{array}$ \\
\hline $\begin{array}{l}\text { Aspiraciones } \\
\text { Emprendedoras }\end{array}$ & $\begin{array}{l}\text { Innovación de productos } \\
\text { Innovación de procesos } \\
\text { Alto crecimiento } \\
\text { Internacionalización } \\
\text { Capital de riesgo }\end{array}$ & $\begin{array}{ll}0,71 & (1) \\
0,16 & (12) \\
0,14 & (13) \\
0,05 & (14) \\
0,16 & (11)\end{array}$ & 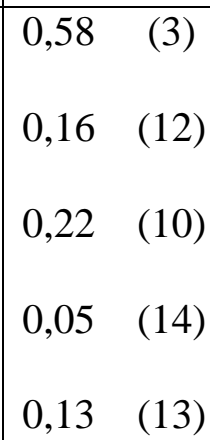 \\
\hline
\end{tabular}

Mínimo 0, Máximo 1. El valor entre paréntesis representa la posición entre los 14 pilares (1 mejor, 14 peor). Fuente: GEDI 2014, GEDI 2015, Ecuador. Elaborado por los autores.

Como se puede notar, los pilares percepción de oportunidades y habilidades para iniciar empresas, son los dos factores donde Ecuador ha obtenido mejores resultados. En el primer 
caso, el buen resultado es una combinación de las oportunidades identificadas por los individuos (GEM), así como el tamaño de mercado.

El pilar de aceptación del riesgo combina actitudes individuales respecto al riesgo, así como riesgo de negocios (legal, económico, tributario, etc.). El apoyo cultural refiere al estatus del emprendimiento, en términos de ser aceptado como una carrera.

El pilar de Emprendimiento por Oportunidad combina la motivación por oportunidad, pero que enfrenta limitaciones por regulación (menor libertad para hacer negocios) De forma similar, la capacidad de absorción tecnológica a nivel de empresa, es uno de los grandes desafíos para el ecosistema emprendedor ecuatoriano, por su relevancia para crear firmas innovadoras y con potencial de crecimiento. Otro pilar con buena evolución es capital humano, que analiza la educación declarada por el emprendedor con los gastos en formación y capacitación de las empresas.

La innovación de productos, innovación de procesos, alto crecimiento, internacionalización y capital de riesgo, estos son pilares claves para lograr emprendimientos de calidad. Este desafío se nota aún más en el pilar de Innovación de Procesos, que se ubica consistentemente entre los 3 peores de los catorce pilares. En este caso, juega un rol importante la poca capacidad de transferencia tecnológica, así como la limitada inversión en I+D. Dos factores cruciales para impulsar el rol del emprendimiento en el desarrollo productivo son internacionalización y alto crecimiento. Ambos se encuentran entre los peores situados en los dos últimos años.

Estos dos pilares tienen una clara orientación estratégica: ampliar mercado en tamaño y en diversificación geográfica. Aquí hay claramente mucho espacio para diseñar políticas 
integrales para que los emprendimientos pequeños y locales, puedan dejar de serlo en un mediano plazo. Los esfuerzos que realizan empresarios, sectores públicos y emprendedores en términos de obtener financiamiento han sido claves para la mejora del pilar de capital de riesgo, hay mucho que hacer aún en términos de desarrollo de mercado de capitales, y creación de instancias de financiamiento para todo el ciclo de vida del emprendimiento.

\section{Relación emprendimiento y desarrollo económico}

Una vez establecidas las características del emprendedor, así como los factores que influyen en el emprendimiento, se analiza la relación del emprendimiento con el desarrollo económico.

En principio, parece existir un amplio consenso con respecto a que la clave para conseguir crecimiento económico y mejoras en la productividad, se encuentra en la capacidad empresarial de una economía. Audretsch (2007) señala las diferencias entre información y conocimiento, considerando qué información se refiere a los hechos que pueden ser codificados, mientras que el conocimiento implica ideas tácitas que no sólo impiden la codificación sino que su valor económico es en gran medida desconocido y asimétrico, lo que podría generar altos costes de transacción, y en general, que la creación de empresas representa el motor de las economías en los países desarrollados (García, 2010). Algunos autores han expuesto diferentes propuestas para definir este proceso. Así, Wennekers, S. y Thurik, R. (1999) establecieron un modelo conceptual en tres niveles: individual, empresarial y macro.

Cienc. Soc. y Econ. UTEQ. 2018. 2(1): 1-22 12 
Más recientemente, Galindo (2014) plantea un modelo en el que se puede comprobar cómo las circunstancias socioeconómicas, la cultura y la evolución del país, afectan a la capacidad emprendedora.

En el Ecuador, a partir de la promulgación de la Carta Magna en el año 2008, se inició una reestructuración de todo el marco legal en coherencia con el Plan Nacional del Buen Vivir, en el que se definen los principios del "nuevo" régimen de desarrollo, el mismo que propone una estrategia a largo plazo para transformar al país en exportador de bioconocimiento y productos con alto valor agregado, estableciendo políticas que impulsen el crecimiento y mejora del nivel de competitividad del sector microempresarial; y, la generación de nuevos emprendimientos innovadores (SENPLADES, 2012).

Existen programas y políticas públicas tales como: EmprendEcuador e InnovaEcuador impulsados por el Ministerio de Coordinación de la Producción, Empleo y Competitividad MCPEC, el Fondo Concursable "Cucayo" de la Secretaria Nacional del Migrante SENAMI, la creación de Centros de Desarrollo Empresarial (CEDE) a nivel nacional del Ministerio de Industrias y Productividad (MIPRO); la política económica y social entre la Organización de las Naciones Unidas y el Ministerio de Inclusión Social para el apoyo a mujeres emprendedoras, así como otras iniciativas generadas desde el sector privado como Siembra Futuro de la Cervecería Nacional y Emprendefe de la Fundación CRISFE del Banco del Pichincha, sin embargo, estas iniciativas han sido aisladas y no han logrado sostenibilidad, lo que no ha permitido la consolidación de los emprendimientos.

No solo es suficiente capacitar, guiar y formar empresarios, para lo cual es necesario que existan verdaderas escuelas de negocios. En este sentido, Guitierrez (2012) menciona que 
para un verdadero desarrollo emprendedor en el país es necesario focalizar esfuerzos y realizar un trabajo integrado entre el gobierno, la empresa privada y la academia, para que el mediano plazo comience a exhibirse los frutos esperados.

El Proyecto Doing Business proporciona una medición objetiva de las normas que regulan la actividad empresarial, analiza y compara las normas que regulan las actividades de las pequeñas y medianas empresas locales a lo largo de su ciclo de vida. El informe cubre diez grupos de indicadores en 185 economías (Banco, 2014).

En el cuadro 2, se detallan las cuatro partes del ciclo de vida y las áreas de Doing Business que corresponden a constitución, expansión actividad e insolvencia. Esta medida muestra lo cerca que cada economía está respecto a las mejores prácticas mundiales en la regulación empresarial. Una puntuación más alta indica un entorno empresarial más eficiente e instituciones legales más fuertes. Ecuador tiene una puntuación de 114 en Facilidad de hacer negocios.

Tabla 2.

Facilidad para hacer Negocios en Ecuador

\begin{tabular}{|l|lr|l|l|l|}
\hline CICLO DE & ÁREAS & QUE & VARIABLES POR & Doing & Doing \\
VIDA & ANALIZA & ÁREA & Business 2014 & Business 2015 \\
& & Clasificación & Clasificación \\
\hline Constitución & Apertura & de & $\begin{array}{l}\text { Requisitos de capital } \\
\text { mínimo } \\
\text { Procedimientos, } \\
\text { empo y costo. }\end{array}$ & 176 & 162 \\
\hline Expansión & Registro & de & Procedimiento & 91 & 69 \\
\hline
\end{tabular}




\begin{tabular}{|c|c|c|c|c|}
\hline & $\begin{array}{l}\text { propiedades } \\
\text { Obtención de } \\
\text { crédito } \\
\text { Protección de } \\
\text { inversiones } \\
\text { Cumplimiento } \\
\text { de contratos }\end{array}$ & $\begin{array}{l}\text { tiempo y costo } \\
\text { Sistema de } \\
\text { información } \\
\text { crediticia } \\
\text { Leyes sobre } \\
\text { garantías } \\
\text { constituidas sobre } \\
\text { bienes inmuebles } \\
\text { Divulgación } \\
\text { responsabilidad en } \\
\text { transacciones entre } \\
\text { partes vinculadas. } \\
\text { Procedimiento, } \\
\text { tiempo y costo de } \\
\text { resolución de una } \\
\text { disputa comercial }\end{array}$ & 138 & 114 \\
\hline Actividad & $\begin{array}{l}\text { Manejo de } \\
\text { permisos de } \\
\text { construcción. } \\
\text { Obtención de } \\
\text { electricidad. } \\
\text { Pago de } \\
\text { impuestos } \\
\text { Comercio } \\
\text { transfronterizo } \\
\text { Empleo } \\
\text { trabajadores }\end{array}$ & $\begin{array}{l}\text { Procedimientos, } \\
\text { tiempo y costo } \\
\text { Procedimiento } \\
\text { tiempo y costo } \\
\text { Pagos, tiempo y tasa } \\
\text { de impuesto total } \\
\text { Documentos, tiempo } \\
\text { y costo. }\end{array}$ & $\begin{array}{l}64 \\
138 \\
91 \\
122\end{array}$ & $\begin{array}{l}69 \\
93\end{array}$ \\
\hline Insolvencia & Resolución de la & Tiempo, costo y tasa & 143 & 148 \\
\hline
\end{tabular}

Cienc. Soc. y Econ. UTEQ. 2018. 2(1): 1-22 15 
\begin{tabular}{|l|l|l|l|l|}
\hline & insolvencia & de recuperación. & & \\
\hline
\end{tabular}

Fuente: Índice Doing Business 2014 y 2015. Elaborado: Autores

No todos los componentes mostrados tienen igual grado de importancia, a pesar que todos influyen de cierta manera en una empresa naciente, es conveniente centrar el análisis en los criterios de apertura de un negocio, obtención de crédito y protección de las inversiones, que son los tres aspectos de mayor gestión a la hora de establecer un emprendimiento.

Como se observa en el cuadro 2, facilidad que tiene Ecuador para hacer negocios, se encuentra en el puesto 162 en lo que es "Apertura de negocios", de los 185 países estudiados, tiene una influencia alta en la manera de cómo lograr un mejor ambiente para la creación de negocios; en cuanto al tiempo, el número de trámites y el costo de los mismos se constituyen un obstáculo para la formalidad y es una de las principales razones para la informalidad. Por su parte, (Andrade, 2012), ha identificado 13 trámites para empezar un negocio de manera formal en Ecuador, estando por encima del promedio regional (9 trámites), en cuanto al número total de días necesarios para inscribir una empresa se requiere 56 días, mientras que otros países podría ser cuestión de muy pocos días, a nivel regional también estamos por encima del promedio (53 días), que para este caso y el anterior sigue siendo un aspecto negativo.

Por otro lado el costo necesario para cubrir con todos los trámites, que incluye todas las tarifas oficiales y los honorarios por servicios legales o profesionales es bastante alto en 
relación a los países de la OCDE, siendo la cantidad de dinero necesaria de $\$ 3.315$ Insumos \$2,010 (61\%) y trámites \$ $1.305(39 \%)$.

Por parte de la obtención de crédito (posición 90) se puede observar que el indicador se origina en la baja credibilidad que le da el gobierno a la información de burós crediticios, derechos legales e información en general.

Los índices antes mencionados son algunos de los obstáculos para constituir empresas formales, siendo mayor número de empresas informales, éstas son empresas (unidades económicas) que pertenecen a los hogares y que no están constituidas en sociedad.

En concordancia con esta definición, se presenta los indicadores de empleo en el sector formal e informal: el primero lo componen las personas que trabajan en establecimientos que tienen RUC; el segundo lo componen las personas ocupadas que trabajan en establecimientos que no se encuentran registrados ante la autoridad tributaria, es decir, las empresas que no tienen Registro Único de Contribuyente (RUC).

El Instituto Nacional de Estadística y Censo en Ecuador, realiza Encuesta de Empleo, Desempleo y Subempleo, como se puede observar en las figuras 3 y 4, la mayoría del empleo adecuado está en el sector formal en el año 2014 el 78\%, es decir perciben ingresos laborales iguales o superiores al salario mínimo, y trabajan igual o más de 40 horas semanales, independientemente del deseo y disponibilidad de trabajar horas adicionales, mientras que para el empleo inadecuado es del $64,4 \%$, lo conforman aquellas personas en empleo, que no satisfacen las condiciones mínimas de horas o ingresos y, que durante la semana de referencia, perciben ingresos laborales menores al salario mínimo y/o trabajan menos de 40 horas a la semana, y pueden o no desear y estar disponibles para trabajar horas 
adicionales. Por su parte, el empleo adecuado en el sector formal en 2015 fue de 77,7\% mientras que el empleo inadecuado en el sector informal fue de 62,8\%. Entre ellos se encuentra el subempleo por insuficiencia de ingresos y por insuficiencia de tiempo de trabajo.

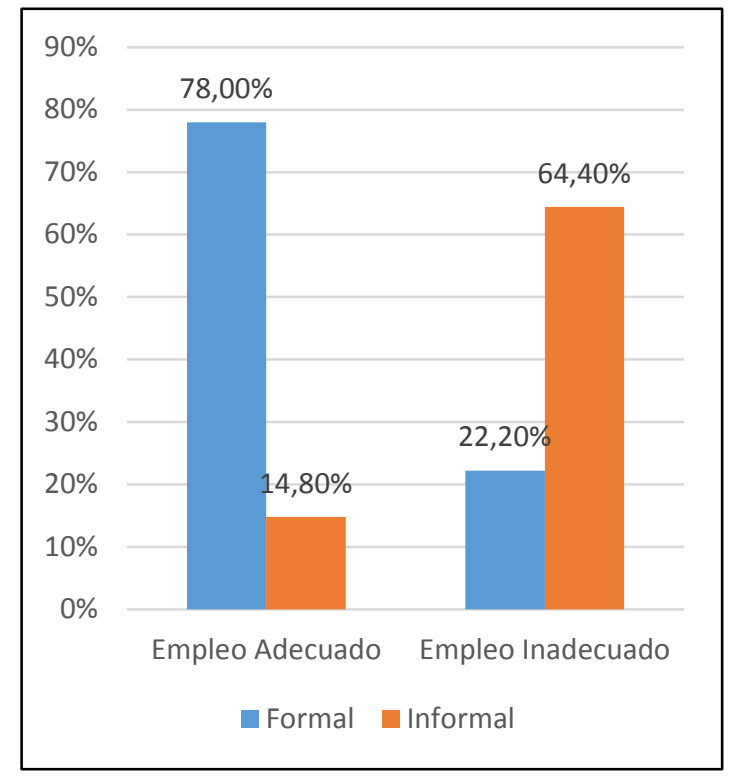

Figura 3. Empleo adecuado e inadecuado en el en el sector formal e informal año 2014. Fuente: ENEMDU, 2014.

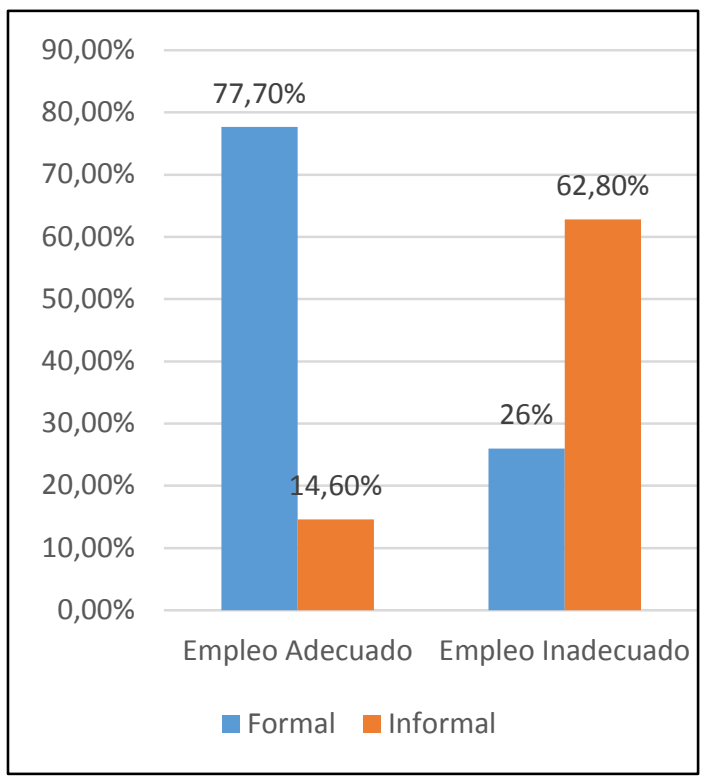

Figura 4. Empleo adecuado e inadecuado sector formal e informal año 2015. Fuente: ENEMDU, 2015.

Estamos claros que para poder empujar la informalidad hacia lo formal de la economía se deben crear políticas y asegurar mejores condiciones laborales, y de alguna $\mathrm{u}$ otra manera promover la protección social a esta economía, los emprendimientos informales no generaron las suficientes fuentes de empleos adecuados, la percepción de los emprendedores respecto al crecimiento empresarial es poco ambiciosa, debido a que las condiciones aun no son lo suficientemente favorables para impulsar a los nuevos negocios. 
Según el Instituto Nacional de Estadísticas y Censos (INEC), hasta el año pasado existían en el Directorio de Empresas y Establecimientos (DIEE) 704.556 empresas, de las cuales el $89,6 \%$ corresponden a microempresas, el 8,2\% pequeña, el 1,7\% mediana y el $0,5 \%$ grandes empresas.

En Ecuador existe una tendencia favorable hacia el emprendimiento gracias al apoyo cultural que existe en nuestro país. Este apoyo se refleja por: el deseo de iniciar un negocio como una opción de carrera 77,8\%; el reconocimiento de estatus $66,1 \%$, respeto a los emprendedores exitosos, y la frecuencia con la que los medios de comunicación transmiten historias de nuevos negocios exitosos 54,1\% (GEM, 2015).

\section{Conclusiones}

De acuerdo al análisis realizado, se ha determinado que debe haber funcionamiento articulado del triángulo empresa privada, estado y universidad; para ello es clave un proceso de diálogo en donde a través de consensos se definan líneas de interés mutuo sobre el apoyo al emprendimiento nacional, es importante para el desarrollo económico del país. El rol de las universidades es un apoyo importante para este desarrollo.

Desde las aulas se deben generar conocimientos en emprendimientos y priorizar la investigación para que estos emprendimientos sobrevivan a largo plazo. Ecuador es un país que tiene alto índice de crecimiento en emprendimiento, sin embargo el cierre de negocios se observa en un elevado porcentaje, esto se debe a las políticas públicas y de créditos, no son las más competitivas para el crecimiento del emprendimiento. Se identificaron 13 trámites para el inicio de un negocio y a un coste elevado. Los requisitos exigidos para la 
creación de empresas formales impiden que los negocios se legalicen, quedando como negocios informales. Esto implica el aumento de la tasa de subempleo, o el empleo inadecuado, es decir, personas que ganan un sueldo menor al básico, y que no se encuentran afiliadas a la seguridad social.

Según el estudio del GEM, para impulsar el emprendimiento en el país, se requiere, entre otras cosas lo siguiente: mejorar condiciones de entorno y financiamiento; generar espacios para la innovación y desarrollo de mercados; promover la vocación y competencias emprendedores y propiciar una mayor conexión entre el mundo académico y el laboral; promover redes de emprendedores; y, fomentar el uso de nuevas tecnologías de la información.

La formación de emprendedores ya no es empírica, ahora forma parte de las carreras profesionales que ofrecen algunas universidades del Ecuador. La carrera de Ingeniería de Emprendedores es una opción que tienen los estudiantes para ingresar con herramientas propias al mercado laboral y demostrar sus cualidades en los negocios. Se suman los centros de emprendedores y las incubadoras de negocios, que también se promocionan en Quito, Guayaquil, Cuenca, Riobamba y Loja.

\section{Referencias Bibliográficas}

Andrade, X. (2012). Las trabas al emprendimiento en Ecuador. Retos, Quito.

Audretsch, D. \&. (2007). The teory of knowledge spilllover entrepreneurship. Journal of Management Studies, 1242-1254.

Cienc. Soc. y Econ. UTEQ. 2018. 2(1): 1-22 20 
Audretsch, D. \&. (2007). The theory of knowledge spillover entrepreneurship. . Journal of Management Studies, 1242-1254.

Banco, M. (2014). Banco Mundial. Doing Business. Midiendo Regulaciones para hacer negocios. Washington, DC.

Baumol, W. (1990). Entrepreneurship: Productive, Unproductive, and Destructive. Journal of Political Economy, 893-921.

Drucker, P. (1997). Innovation and Entrepreneurship: Practice and Principles. Londres: Heinemann.

ENEMDU. (2016, Marzo). Encuesta Nacional de Empleo, Desempleo y Subempleo. Ecuador: Ecuador en cifras.

Galindo, M. \&. (2014). La actividad emprendedora y competitividad: factores que inciden los emprendedores. Revistas Científicas Complutenses, 66-69.

García, A. (2010). Actividad emprendedora e indsutria creativa. Economistas , 69-78.

GEDI. (2014-2015). Global Entrepreneurship Development Institute. Ecuador.

GEM. (2015). Global Entrepreneurship Monitor: reporte anual Ecuador 2015. Lasio V, Caicedo G, Ordeñana X, Izquierdo E. Ecuador : Escuela Superior Politécnica del Litoral, La Primera Escuela de Negocios del Ecuador. Ediciones Uniandes.

Guitierrez - Solana, F. (2013). Conocimiento, Creatividad, Emprendimiento e Innovación. Revista Tribuna Complutense, 4.

Guitierrez, G. (2012). El fenómeno del emprendedurismo en el Salvador: comparativa con paises GEM. Revista Retos, 17-36. 
Herrera, C. (2012). Una investigación en emprendimiento: Caracterización Hisrich, D.R.; Peters, P.M.; Dean, S.A. (2005). Interamericana de España.

Hisrich, P.; Peters, M.P; Shepherd, D.A. (2005). Entrepreneurship. Interamericana.

Lederma Daniel, J. M. (2014). El emprendimiento en América Latina: muchas empresas y poca innovación. Washington: Creative Commons.

Lorca, L. (2013). Modelo de Factores que inciden en el éxito de emprendimientos sociales en América Latina: Estudio Cualitativo. Chile.

Moreno, L. A. (2009). Aumentando la competitividad global a través de la integracion: retos y oportunidades para América Latina y el Caribe. Revista de Economía, 17-25.

Ramírez, J. (2009). PYMES más competitivas. Bogotá: Ediciones S.A., Bogotá.

Reynolds, P. (2005). Global Entrepreneurship Monitor: Data Collection Desgin and Implementation 1998-2003. Small Bussiness Economics, 24, 205-231.

Schumpeter, J. (1982). Historia del Análisis Económico. Arial.

SENPLADES. (2012). Secretaria Nacional de Planificación y Desarrollo. Plan Nacional Buen Vivir 2013-2017. Folleto Informativo. Transformacion de la Matriz Productiva. Quito.

Varela, R. (2001). La cultura empresarial como Estrategia de desarrollo para América Latina. Revista Icesi- Universidad ICESI.

Wennekers, S. y Thurik, R. (1999). Linking Entrepreneurship and Economic Growth. Small Business Economics, 27-55. 